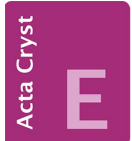

CRYSTALLOGRAPHIC COMMUNICATIONS

ISSN 2056-9890

\section{Crystal structure of dimethomorph}

\author{
Gihaeng Kang, Jineun Kim,* Eunjin Kwon and Tae Ho \\ Kim*
}

Department of Chemistry and Research Institute of Natural Sciences, Gyeongsang National University, Jinju 660-701, Republic of Korea. *Correspondence e-mail:

thkim@gnu.ac.kr, jekim@gnu.ac.kr

Received 2 August 2015; accepted 6 August 2015

Edited by P. C. Healy, Griffith University, Australia

In the title compound, $\mathrm{C}_{21} \mathrm{H}_{22} \mathrm{ClNO}_{4}$ [systematic name: $(E)-3$ (4-chlorophenyl)-3-(3,4-dimethoxyphenyl)-1-(morpholin-4-yl)prop-2-en-1-one], which is the morpholine fungicide dimethomorph, the dihedral angles between the mean planes of the central chlorophenyl and the terminal benzene and morpholine (r.m.s. deviation $=0.2233 \AA$ ) rings are $71.74(6)$ and $63.65(7)^{\circ}$, respectively. In the crystal, molecules are linked via $\mathrm{C}-\mathrm{H} \cdots \mathrm{O}$ hydrogen bonds and weak $\mathrm{Cl} \cdots \pi$ interactions [3.8539 (11) $]$ ], forming a three-dimensional structure.

Keywords: crystal structure; dimethomorph; prop-2-en-1-one; fungicide.

CCDC reference: 1417163

\section{Related literature}

For information on the fungicidal properties of the title compound, see: Xu et al. (2015). For related crystal structures, see: Chai \& Liu (2011); Lu \& Shi (2011).

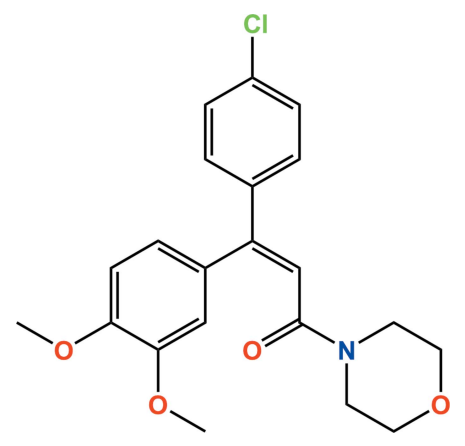

Monoclinic, $P 2_{1} / c$

$a=6.6238(2) \AA$

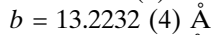

$c=21.4810(7) \AA$

$\beta=97.1674(19)^{\circ}$

$V=1866.77(10) \AA^{3}$

\subsection{Data collection}

Bruker APEXII CCD diffractometer

Absorption correction: multi-scan

(SADABS; Bruker, 2013)

$T_{\min }=0.917, T_{\max }=0.993$

\subsection{Refinement}

$R\left[F^{2}>2 \sigma\left(F^{2}\right)\right]=0.048$

$w R\left(F^{2}\right)=0.133$

$S=1.04$

4276 reflections
$Z=4$

Mo $K \alpha$ radiation

$\mu=0.23 \mathrm{~mm}^{-1}$

$T=173 \mathrm{~K}$

$0.38 \times 0.06 \times 0.03 \mathrm{~mm}$

Table 1

Hydrogen-bond geometry $\left(\AA{ }^{\circ}\right)$.

\begin{tabular}{lllll}
\hline$D-\mathrm{H} \cdots A$ & $D-\mathrm{H}$ & $\mathrm{H} \cdots A$ & $D \cdots A$ & $D-\mathrm{H} \cdots A$ \\
\hline $\mathrm{C} 1-\mathrm{H} 1 B \cdots \mathrm{O} 2^{\mathrm{i}}$ & 0.99 & 2.53 & $3.167(2)$ & 122 \\
$\mathrm{C} 13-\mathrm{H} 13 \cdots \mathrm{O} 2^{\mathrm{ii}}$ & 0.95 & 2.38 & $3.166(2)$ & 140 \\
$\mathrm{C} 20-\mathrm{H} 20 B \cdots \mathrm{O} 1^{\mathrm{iii}}$ & 0.98 & 2.64 & $3.010(2)$ & 103 \\
\hline
\end{tabular}

Symmetry codes: (i) $x+1, y, z$; (ii) $-x, y+\frac{1}{2},-z+\frac{3}{2}$; (iii) $-x+1,-y+1,-z+2$.

Data collection: APEX2 (Bruker, 2013); cell refinement: SAINT (Bruker, 2013); data reduction: SAINT; program(s) used to solve structure: SHELXS97 (Sheldrick, 2008); program(s) used to refine structure: SHELXL2013 (Sheldrick, 2015); molecular graphics: DIAMOND (Brandenburg, 2010); software used to prepare material for publication: SHELXTL (Sheldrick, 2008).

\title{
Acknowledgements
}

This research was supported by the Basic Science Research Program through the National Research Foundation of Korea (NRF) funded by the Ministry of Education, Science and Technology (No. 2015R1D1A4A01020317).

Supporting information for this paper is available from the IUCr electronic archives (Reference: HG5456).

\section{References}

Brandenburg, K. (2010). DIAMOND. Crystal Impact GbR, Bonn, Germany. Bruker (2013). APEX2, SAINT and SADABS. Bruker AXS Inc., Madison, Wisconsin, USA.

Chai, B. \& Liu, C. (2011). Acta Cryst. E67, o1780.

Lu, B.-L. \& Shi, M. (2011). Chem. Eur. J. 17, 9070-9075.

Sheldrick, G. M. (2008). Acta Cryst. A64, 112-122.

Sheldrick, G. M. (2015). Acta Cryst. C71, 3-8.

Xu, X., Liang, S., Meng, X., Zhang, M., Chen, Y., Zhao, D. \& Li, Y. (2015). J. Chromatogr. B, 988, 182-186.

\section{Experimental}

2.1. Crystal data

$\mathrm{C}_{21} \mathrm{H}_{22} \mathrm{ClNO}_{4}$

$M_{r}=387.84$ 


\section{supporting information}

Acta Cryst. (2015). E71, o654 [https://doi.org/10.1107/S2056989015014735]

\section{Crystal structure of dimethomorph}

\section{Gihaeng Kang, Jineun Kim, Eunjin Kwon and Tae Ho Kim}

\section{S1. Comment}

Dimethomorph [systematic name: (E)-3-(4-chlorophenyl)-3-(3,4-dimethoxyphenyl)-1-(morpholin-4-yl)prop-2-en-1-one] is a morpholine fungicide that has been mainly applied on grapevines, apples, ginsengs, tomatoes, potatoes, cucumbers, Chinese cabbage and other crops. (Xu et al., 2015). The dihedral angles between the planes of the central chlorophenyl and the terminal benzene and mean plane [r.m.s. deviation $=0.2233$ ] of morpholine rings are $71.74(6)$ and $63.65(7)^{\circ}$, respectively. All bond lengths and bond angles are normal and comparable to those observed in similar crystal structures (Chai \& Liu, 2011; Lu \& Shi, 2011).

In the crystal structure (Fig. 2), $\mathrm{C}-\mathrm{H} \cdots \mathrm{O}$ hydrogen bonds (Table 1) and weak intermolecular $\mathrm{C} 11-\mathrm{Cl1} \cdots \mathrm{Cg} 1^{\text {iv }}(\mathrm{Cg} 1$ is the centroid of the $\mathrm{C} 8-\mathrm{C} 13 \mathrm{ring}$ ) interaction with a chlorophenyl ring are present, resulting in a three-dimensional network [for symmetry code: (iv), $-x,-y+1,-z+1]$.

\section{S2. Experimental}

The title compound was purchased from the Dr. Ehrenstorfer $\mathrm{GmbH}$ Company. Slow evaporation of a solution in $\mathrm{CH}_{3} \mathrm{OH}$ gave single crystals suitable for X-ray analysis.

\section{S3. Refinement}

All H-atoms were positioned geometrically and refined using a riding model with $\mathrm{d}(\mathrm{C}-\mathrm{H})=0.98 \AA, U_{\text {iso }}=1.5 U_{\text {eq }}(\mathrm{C})$ for methyl group, $\mathrm{d}(\mathrm{C}-\mathrm{H})=0.99 \AA, U_{\text {iso }}=1.2 U_{\text {eq }}(\mathrm{C})$ for $\mathrm{CH}_{2}$ group, $\mathrm{d}(\mathrm{C}-\mathrm{H})=0.95 \AA, U_{\text {iso }}=1.2 U_{\text {eq }}(\mathrm{C})$ for $\mathrm{Csp} p^{2}-\mathrm{H}$ and aromatic $\mathrm{C}-\mathrm{H}$. 


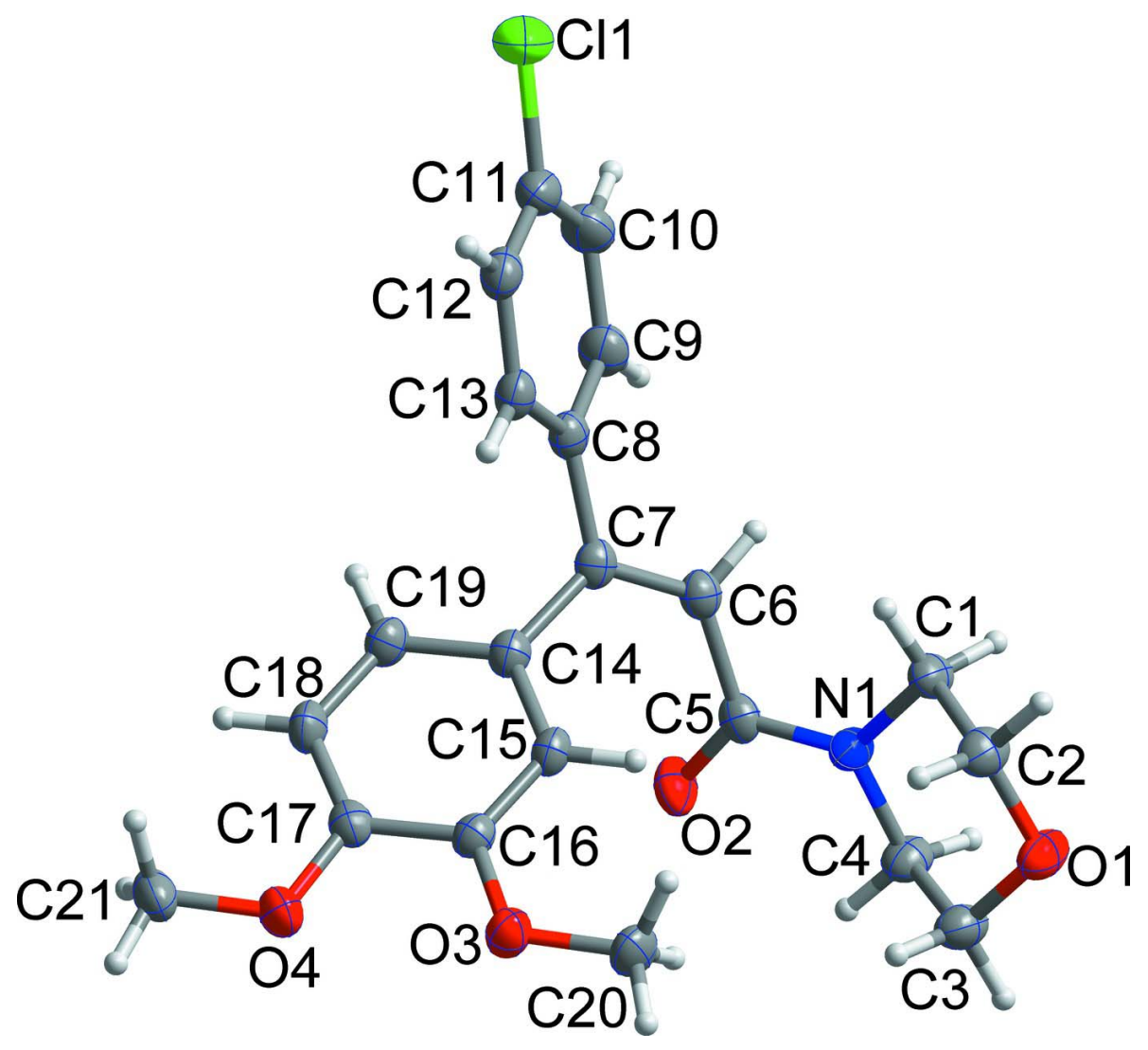

Figure 1

The asymmetric unit of the title compound with the atom numbering scheme. Displacement ellipsoids are drawn at the $50 \%$ probability level. $\mathrm{H}$ atoms are shown as small spheres of arbitrary radius. 


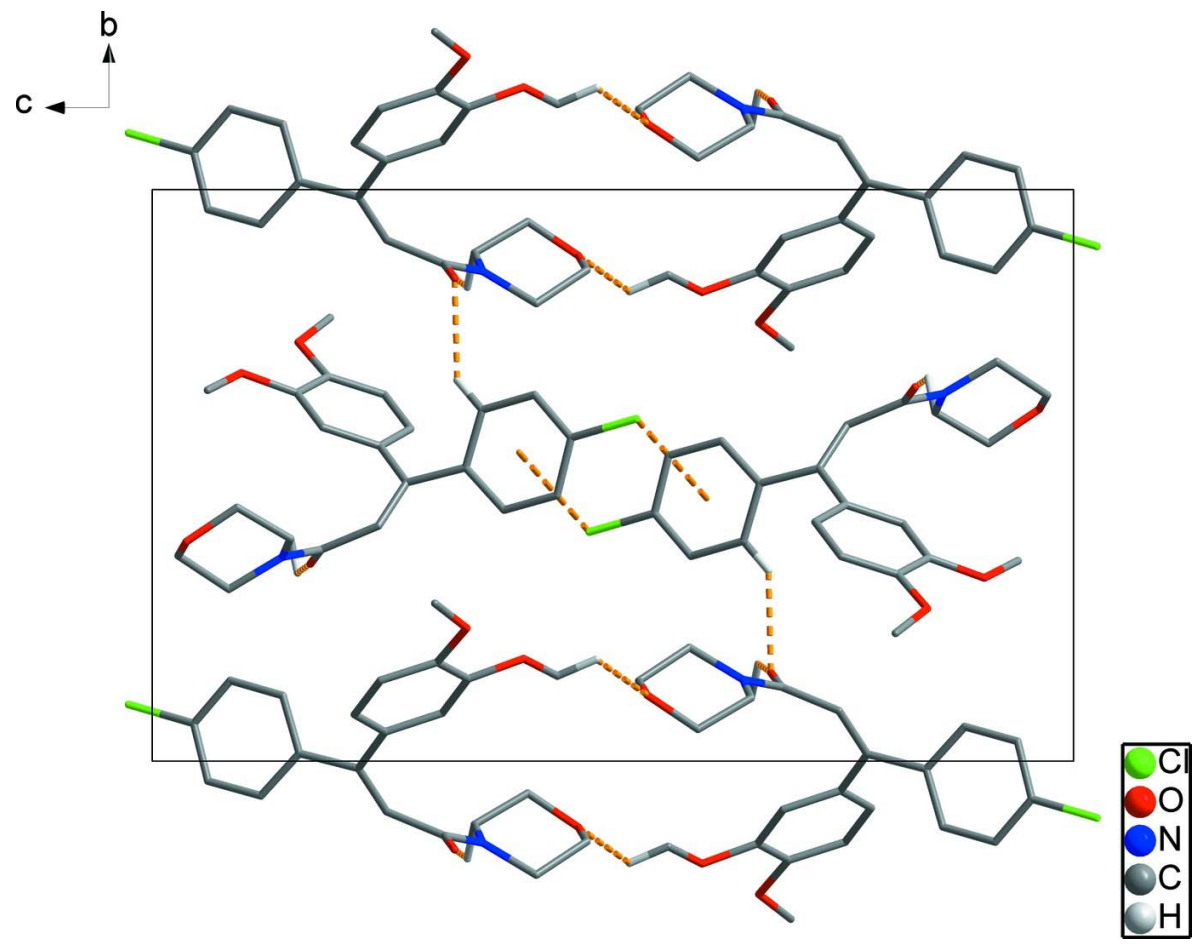

Figure 2

Crystal packing viewed along the $a$ axis. The intermolecular interactions are shown as dashed lines.

(E)-3-(4-Chlorophenyl)-3-(3,4-dimethoxyphenyl)-1-(morpholin-4-yl)prop-2-en-1-one

Crystal data

$\mathrm{C}_{21} \mathrm{H}_{22} \mathrm{ClNO}_{4}$

$M_{r}=387.84$

Monoclinic, $P 2{ }_{1} / c$

$a=6.6238(2) \AA$

$b=13.2232(4) \AA$

$c=21.4810(7) \AA$

$\beta=97.1674(19)^{\circ}$

$V=1866.77(10) \AA^{3}$

$Z=4$

\section{Data collection}

Bruker APEXII CCD

diffractometer

$\varphi$ and $\omega$ scans

Absorption correction: multi-scan

(SADABS; Bruker, 2013)

$T_{\min }=0.917, T_{\max }=0.993$

18090 measured reflections

Refinement

Refinement on $F^{2}$

Least-squares matrix: full

$R\left[F^{2}>2 \sigma\left(F^{2}\right)\right]=0.048$

$w R\left(F^{2}\right)=0.133$
$F(000)=816$

$D_{\mathrm{x}}=1.380 \mathrm{Mg} \mathrm{m}^{-3}$

Mo $K \alpha$ radiation, $\lambda=0.71073 \AA$

Cell parameters from 3670 reflections

$\theta=2.5-24.0^{\circ}$

$\mu=0.23 \mathrm{~mm}^{-1}$

$T=173 \mathrm{~K}$

Needle, colourless

$0.38 \times 0.06 \times 0.03 \mathrm{~mm}$

4276 independent reflections

3119 reflections with $I>2 \sigma(I)$

$R_{\text {int }}=0.047$

$\theta_{\text {max }}=27.5^{\circ}, \theta_{\min }=1.8^{\circ}$

$h=-8 \rightarrow 8$

$k=-14 \rightarrow 17$

$l=-27 \rightarrow 27$

$S=1.04$

4276 reflections

246 parameters

0 restraints 
Hydrogen site location: inferred from neighbouring sites

H-atom parameters constrained

$$
\begin{aligned}
& w=1 /\left[\sigma^{2}\left(F_{\mathrm{o}}^{2}\right)+(0.059 P)^{2}+0.6606 P\right] \\
& \text { where } P=\left(F_{\mathrm{o}}^{2}+2 F_{\mathrm{c}}{ }^{2}\right) / 3 \\
& (\Delta / \sigma)_{\max }<0.001 \\
& \Delta \rho_{\max }=0.27 \mathrm{e} \AA^{-3} \\
& \Delta \rho_{\min }=-0.53 \mathrm{e} \AA^{-3}
\end{aligned}
$$

Special details

Geometry. All e.s.d.'s (except the e.s.d. in the dihedral angle between two 1.s. planes) are estimated using the full

\begin{tabular}{|c|c|c|c|c|}
\hline & $x$ & $y$ & $z$ & $U_{\text {iso }} * / U_{\text {eq }}$ \\
\hline $\mathrm{C} 11$ & $0.31366(10)$ & $0.59912(5)$ & $0.47448(3)$ & $0.0574(2)$ \\
\hline O1 & $0.5956(2)$ & $0.38430(11)$ & $0.96279(6)$ & $0.0370(4)$ \\
\hline $\mathrm{O} 2$ & $-0.0418(2)$ & $0.34526(10)$ & $0.82830(7)$ & $0.0370(4)$ \\
\hline $\mathrm{O} 3$ & $-0.1413(2)$ & $0.67931(10)$ & $0.90317(6)$ & $0.0337(3)$ \\
\hline $\mathrm{O} 4$ & -0.4849 (2) & $0.73290(10)$ & $0.84142(6)$ & $0.0368(4)$ \\
\hline N1 & $0.2962(2)$ & $0.35718(12)$ & $0.85873(7)$ & $0.0281(4)$ \\
\hline $\mathrm{C} 1$ & 0.5023 & $0.38939(15)$ & $0.84994(9)$ & $0.0305(4)$ \\
\hline H1A & 0.4969 & 0.4347 & 0.8131 & $0.037 *$ \\
\hline H1B & 0.5855 & 0.3296 & 0.8421 & $0.037^{*}$ \\
\hline $\mathrm{C} 2$ & $0.5976(3)$ & $0.44402(16)$ & $0.90772(9)$ & $0.0347(5)$ \\
\hline $\mathrm{H} 2 \mathrm{~A}$ & 0.7399 & 0.4617 & 0.9027 & $0.042 *$ \\
\hline $\mathrm{H} 2 \mathrm{~B}$ & 0.5226 & 0.5078 & 0.9125 & $0.042^{*}$ \\
\hline $\mathrm{C} 3$ & 0.3918 & $0.35949(17)$ & $0.97178(9)$ & $0.0364(5)$ \\
\hline $\mathrm{H} 3 \mathrm{~A}$ & 0.3150 & 0.4224 & 0.9772 & $0.044 *$ \\
\hline H3B & 0.3928 & 0.3187 & 1.0104 & $0.044 *$ \\
\hline $\mathrm{C} 4$ & $0.2872(3)$ & $0.30099(16)$ & $0.91686(9)$ & $0.0335(5)$ \\
\hline $\mathrm{H} 4 \mathrm{~A}$ & 0.3542 & 0.2345 & 0.9142 & $0.040 *$ \\
\hline H4B & 0.1434 & 0.2890 & 0.9229 & $0.040 *$ \\
\hline $\mathrm{C} 5$ & $0.1286(3)$ & $0.37008(13)$ & $0.81674(9)$ & $0.0267(4)$ \\
\hline C6 & $0.1627(3)$ & $0.41177(14)$ & $0.75448(9)$ & $0.0277(4)$ \\
\hline H6 & 0.2683 & 0.3819 & 0.7346 & $0.033^{*}$ \\
\hline $\mathrm{C} 7$ & $0.0584(3)$ & $0.48755(13)$ & $0.72382(9)$ & $0.0263(4)$ \\
\hline $\mathrm{C} 8$ & $0.1046(3)$ & $0.51370(14)$ & $0.65975(9)$ & $0.0274(4)$ \\
\hline C9 & $0.1513(3)$ & $0.43952(16)$ & $0.61797(9)$ & $0.0354(5)$ \\
\hline H9 & 0.1412 & 0.3702 & 0.6289 & $0.043^{*}$ \\
\hline $\mathrm{C} 10$ & 0.2124 & $0.46527(17)$ & $0.56054(10)$ & $0.0411(5)$ \\
\hline $\mathrm{H} 10$ & 0.2424 & 0.4140 & 0.5321 & $0.049 *$ \\
\hline C11 & $0.2291(3)$ & $0.56604(18)$ & $0.54519(10)$ & $0.0378(5)$ \\
\hline $\mathrm{C} 12$ & $0.1811(3)$ & $0.64122(16)$ & $0.58492(10)$ & $0.0350(5)$ \\
\hline H12 & 0.1923 & 0.7103 & 0.5737 & $0.042 *$ \\
\hline $\mathrm{C} 13$ & 0.1159 & $0.61476(15)$ & $0.64179(9)$ & $0.0303(4)$ \\
\hline H13 & 0.0785 & 0.6664 & 0.6689 & $0.036^{*}$ \\
\hline C14 & $-0.0896(3)$ & $0.55131(13)$ & $0.75275(9)$ & $0.0261(4)$ \\
\hline C15 & -0.0445 & $0.58425(13)$ & $0.81488(8)$ & $0.0254(4)$ \\
\hline
\end{tabular}
covariance matrix. The cell e.s.d.'s are taken into account individually in the estimation of e.s.d.'s in distances, angles and torsion angles; correlations between e.s.d.'s in cell parameters are only used when they are defined by crystal symmetry. An approximate (isotropic) treatment of cell e.s.d.'s is used for estimating e.s.d.'s involving 1.s. planes.

Fractional atomic coordinates and isotropic or equivalent isotropic displacement parameters $\left(\AA^{2}\right)$ 


$\begin{array}{lllll}\text { H15 } & 0.0808 & 0.5648 & 0.8382 & 0.030^{*} \\ \text { C16 } & -0.1772(3) & 0.64407(13) & 0.84292(8) & 0.0255(4) \\ \text { C17 } & -0.3626(3) & 0.67311(13) & 0.80926(9) & 0.0278(4) \\ \text { C18 } & -0.4093(3) & 0.64144(14) & 0.74781(9) & 0.0304(4) \\ \text { H18 } & -0.5351 & 0.6606 & 0.7247 & 0.037^{*} \\ \text { C19 } & -0.2734(3) & 0.58161(14) & 0.71962(9) & 0.0293(4) \\ \text { H19 } & -0.3067 & 0.5612 & 0.6772 & 0.035^{*} \\ \text { C20 } & 0.0428(3) & 0.64921(17) & 0.93982(9) & 0.0369(5) \\ \text { H20A } & 0.0476 & 0.5753 & 0.9428 & 0.055^{*} \\ \text { H20B } & 0.0485 & 0.6782 & 0.9820 & 0.055^{*} \\ \text { H20C } & 0.1591 & 0.6734 & 0.9199 & 0.055^{*} \\ \text { C21 } & -0.6601(3) & 0.77604(16) & 0.80687(10) & 0.0388(5) \\ \text { H21A } & -0.6192 & 0.8187 & 0.7733 & 0.058^{*} \\ \text { H21B } & -0.7324 & 0.8172 & 0.8349 & 0.058^{*} \\ \text { H21C } & -0.7499 & 0.7220 & 0.7885 & 0.058^{*} \\ \end{array}$

Atomic displacement parameters $\left(\AA^{2}\right)$

\begin{tabular}{lllllll}
\hline & $U^{11}$ & $U^{22}$ & $U^{33}$ & $U^{12}$ & $U^{13}$ & $U^{23}$ \\
\hline C11 & $0.0525(4)$ & $0.0813(5)$ & $0.0412(3)$ & $0.0133(3)$ & $0.0173(3)$ & $0.0147(3)$ \\
O1 & $0.0326(8)$ & $0.0484(8)$ & $0.0281(7)$ & $-0.0095(7)$ & $-0.0041(6)$ & $0.0043(6)$ \\
O2 & $0.0226(7)$ & $0.0352(7)$ & $0.0529(9)$ & $-0.0019(6)$ & $0.0031(6)$ & $0.0124(7)$ \\
O3 & $0.0356(8)$ & $0.0358(7)$ & $0.0283(7)$ & $0.0058(6)$ & $-0.0015(6)$ & $-0.0037(6)$ \\
O4 & $0.0333(8)$ & $0.0410(8)$ & $0.0362(8)$ & $0.0113(6)$ & $0.0048(6)$ & $0.0003(6)$ \\
N1 & $0.0243(8)$ & $0.0340(9)$ & $0.0255(8)$ & $-0.0049(7)$ & $0.0017(7)$ & $0.0040(7)$ \\
C1 & $0.0235(10)$ & $0.0395(11)$ & $0.0283(10)$ & $-0.0039(8)$ & $0.0028(8)$ & $0.0010(9)$ \\
C2 & $0.0310(11)$ & $0.0399(11)$ & $0.0325(11)$ & $-0.0102(9)$ & $0.0011(9)$ & $0.0024(9)$ \\
C3 & $0.0363(12)$ & $0.0462(12)$ & $0.0270(10)$ & $-0.0058(10)$ & $0.0045(9)$ & $0.0025(9)$ \\
C4 & $0.0324(11)$ & $0.0373(11)$ & $0.0309(11)$ & $-0.0074(9)$ & $0.0045(9)$ & $0.0065(9)$ \\
C5 & $0.0241(9)$ & $0.0211(9)$ & $0.0346(10)$ & $0.0003(7)$ & $0.0028(8)$ & $-0.0010(8)$ \\
C6 & $0.0238(9)$ & $0.0271(9)$ & $0.0313(10)$ & $-0.0006(8)$ & $-0.0001(8)$ & $-0.0017(8)$ \\
C7 & $0.0236(9)$ & $0.0252(9)$ & $0.0285(10)$ & $-0.0032(8)$ & $-0.0027(8)$ & $-0.0029(8)$ \\
C8 & $0.0228(9)$ & $0.0302(10)$ & $0.0279(10)$ & $0.0004(8)$ & $-0.0020(8)$ & $-0.0016(8)$ \\
C9 & $0.0378(12)$ & $0.0332(10)$ & $0.0347(11)$ & $0.0031(9)$ & $0.0020(9)$ & $-0.0045(9)$ \\
C10 & $0.0394(12)$ & $0.0484(13)$ & $0.0361(12)$ & $0.0060(10)$ & $0.0077(10)$ & $-0.0077(10)$ \\
C11 & $0.0301(11)$ & $0.0539(13)$ & $0.0298(11)$ & $0.0056(10)$ & $0.0046(9)$ & $0.0057(10)$ \\
C12 & $0.0290(11)$ & $0.0387(11)$ & $0.0360(11)$ & $0.0038(9)$ & $-0.0013(9)$ & $0.0073(9)$ \\
C13 & $0.0279(10)$ & $0.0313(10)$ & $0.0299(10)$ & $0.0036(8)$ & $-0.0031(8)$ & $0.0015(8)$ \\
C14 & $0.0251(9)$ & $0.0232(9)$ & $0.0291(10)$ & $-0.0019(7)$ & $-0.0003(8)$ & $0.0024(8)$ \\
C15 & $0.0236(9)$ & $0.0229(9)$ & $0.0278(10)$ & $-0.0012(7)$ & $-0.0032(8)$ & $0.0029(7)$ \\
C16 & $0.0270(10)$ & $0.0235(9)$ & $0.0253(10)$ & $-0.0024(7)$ & $0.0008(8)$ & $0.0025(7)$ \\
C17 & $0.0250(10)$ & $0.0245(9)$ & $0.0343(11)$ & $-0.0001(8)$ & $0.0051(8)$ & $0.0042(8)$ \\
C18 & $0.0224(9)$ & $0.0326(10)$ & $0.0345(11)$ & $0.0010(8)$ & $-0.0033(8)$ & $0.0043(8)$ \\
C19 & $0.0281(10)$ & $0.0304(10)$ & $0.0279(10)$ & $-0.0015(8)$ & $-0.0024(8)$ & $-0.0010(8)$ \\
C20 & $0.0337(11)$ & $0.0473(12)$ & $0.0275(11)$ & $0.0019(10)$ & $-0.0055(9)$ & $-0.0033(9)$ \\
C21 & $0.0278(10)$ & $0.0437(12)$ & $0.0458(13)$ & $0.0082(9)$ & $0.0084(9)$ & $0.0084(10)$ \\
& & & & & & \\
\hline & & & & & & \\
\hline
\end{tabular}


Geometric parameters $\left(\AA,{ }^{\circ}\right)$

\begin{tabular}{|c|c|c|c|}
\hline $\mathrm{C} 11-\mathrm{C} 11$ & $1.739(2)$ & $\mathrm{C} 8-\mathrm{C} 9$ & $1.390(3)$ \\
\hline $\mathrm{O} 1-\mathrm{C} 2$ & $1.424(2)$ & $\mathrm{C} 8-\mathrm{C} 13$ & $1.395(3)$ \\
\hline $\mathrm{O} 1-\mathrm{C} 3$ & $1.426(2)$ & $\mathrm{C} 9-\mathrm{C} 10$ & $1.388(3)$ \\
\hline $\mathrm{O} 2-\mathrm{C} 5$ & $1.230(2)$ & C9-H9 & 0.9500 \\
\hline $\mathrm{O} 3-\mathrm{C} 16$ & $1.368(2)$ & $\mathrm{C} 10-\mathrm{C} 11$ & $1.380(3)$ \\
\hline $\mathrm{O} 3-\mathrm{C} 20$ & $1.423(2)$ & $\mathrm{C} 10-\mathrm{H} 10$ & 0.9500 \\
\hline $\mathrm{O} 4-\mathrm{C} 17$ & $1.378(2)$ & $\mathrm{C} 11-\mathrm{C} 12$ & $1.373(3)$ \\
\hline $\mathrm{O} 4-\mathrm{C} 21$ & $1.417(2)$ & $\mathrm{C} 12-\mathrm{C} 13$ & $1.390(3)$ \\
\hline $\mathrm{N} 1-\mathrm{C} 5$ & $1.351(2)$ & $\mathrm{C} 12-\mathrm{H} 12$ & 0.9500 \\
\hline $\mathrm{N} 1-\mathrm{C} 4$ & $1.461(2)$ & $\mathrm{C} 13-\mathrm{H} 13$ & 0.9500 \\
\hline $\mathrm{N} 1-\mathrm{C} 1$ & $1.464(2)$ & $\mathrm{C} 14-\mathrm{C} 19$ & $1.390(3)$ \\
\hline $\mathrm{C} 1-\mathrm{C} 2$ & $1.505(3)$ & $\mathrm{C} 14-\mathrm{C} 15$ & $1.400(3)$ \\
\hline $\mathrm{C} 1-\mathrm{H} 1 \mathrm{~A}$ & 0.9900 & $\mathrm{C} 15-\mathrm{C} 16$ & $1.376(3)$ \\
\hline $\mathrm{C} 1-\mathrm{H} 1 \mathrm{~B}$ & 0.9900 & $\mathrm{C} 15-\mathrm{H} 15$ & 0.9500 \\
\hline $\mathrm{C} 2-\mathrm{H} 2 \mathrm{~A}$ & 0.9900 & $\mathrm{C} 16-\mathrm{C} 17$ & $1.398(3)$ \\
\hline $\mathrm{C} 2-\mathrm{H} 2 \mathrm{~B}$ & 0.9900 & $\mathrm{C} 17-\mathrm{C} 18$ & $1.383(3)$ \\
\hline $\mathrm{C} 3-\mathrm{C} 4$ & $1.505(3)$ & $\mathrm{C} 18-\mathrm{C} 19$ & $1.392(3)$ \\
\hline $\mathrm{C} 3-\mathrm{H} 3 \mathrm{~A}$ & 0.9900 & $\mathrm{C} 18-\mathrm{H} 18$ & 0.9500 \\
\hline C $3-\mathrm{H} 3 \mathrm{~B}$ & 0.9900 & C19-H19 & 0.9500 \\
\hline $\mathrm{C} 4-\mathrm{H} 4 \mathrm{~A}$ & 0.9900 & $\mathrm{C} 20-\mathrm{H} 20 \mathrm{~A}$ & 0.9800 \\
\hline $\mathrm{C} 4-\mathrm{H} 4 \mathrm{~B}$ & 0.9900 & $\mathrm{C} 20-\mathrm{H} 20 \mathrm{~B}$ & 0.9800 \\
\hline $\mathrm{C} 5-\mathrm{C} 6$ & $1.490(3)$ & $\mathrm{C} 20-\mathrm{H} 20 \mathrm{C}$ & 0.9800 \\
\hline $\mathrm{C} 6-\mathrm{C} 7$ & $1.342(3)$ & $\mathrm{C} 21-\mathrm{H} 21 \mathrm{~A}$ & 0.9800 \\
\hline C6-H6 & 0.9500 & $\mathrm{C} 21-\mathrm{H} 21 \mathrm{~B}$ & 0.9800 \\
\hline C7-C14 & $1.487(3)$ & $\mathrm{C} 21-\mathrm{H} 21 \mathrm{C}$ & 0.9800 \\
\hline $\mathrm{C} 7-\mathrm{C} 8$ & $1.487(3)$ & & \\
\hline $\mathrm{C} 2-\mathrm{O} 1-\mathrm{C} 3$ & $110.32(15)$ & $\mathrm{C} 8-\mathrm{C} 9-\mathrm{H} 9$ & 119.5 \\
\hline $\mathrm{C} 16-\mathrm{O} 3-\mathrm{C} 20$ & $117.63(15)$ & $\mathrm{C} 11-\mathrm{C} 10-\mathrm{C} 9$ & 119.3 \\
\hline $\mathrm{C} 17-\mathrm{O} 4-\mathrm{C} 21$ & $117.58(15)$ & $\mathrm{C} 11-\mathrm{C} 10-\mathrm{H} 10$ & 120.3 \\
\hline $\mathrm{C} 5-\mathrm{N} 1-\mathrm{C} 4$ & $121.14(16)$ & $\mathrm{C} 9-\mathrm{C} 10-\mathrm{H} 10$ & 120.3 \\
\hline $\mathrm{C} 5-\mathrm{N} 1-\mathrm{C} 1$ & $125.36(16)$ & $\mathrm{C} 12-\mathrm{C} 11-\mathrm{C} 10$ & $121.2(2)$ \\
\hline $\mathrm{C} 4-\mathrm{N} 1-\mathrm{C} 1$ & $113.35(15)$ & $\mathrm{C} 12-\mathrm{C} 11-\mathrm{C} 11$ & $119.04(17)$ \\
\hline $\mathrm{N} 1-\mathrm{C} 1-\mathrm{C} 2$ & $109.58(15)$ & $\mathrm{C} 10-\mathrm{C} 11-\mathrm{C} 11$ & $119.71(17)$ \\
\hline $\mathrm{N} 1-\mathrm{C} 1-\mathrm{H} 1 \mathrm{~A}$ & 109.8 & $\mathrm{C} 11-\mathrm{C} 12-\mathrm{C} 13$ & 119.03 (19) \\
\hline $\mathrm{C} 2-\mathrm{C} 1-\mathrm{H} 1 \mathrm{~A}$ & 109.8 & $\mathrm{C} 11-\mathrm{C} 12-\mathrm{H} 12$ & 120.5 \\
\hline $\mathrm{N} 1-\mathrm{C} 1-\mathrm{H} 1 \mathrm{~B}$ & 109.8 & $\mathrm{C} 13-\mathrm{C} 12-\mathrm{H} 12$ & 120.5 \\
\hline $\mathrm{C} 2-\mathrm{C} 1-\mathrm{H} 1 \mathrm{~B}$ & 109.8 & $\mathrm{C} 12-\mathrm{C} 13-\mathrm{C} 8$ & 121.15 (19) \\
\hline $\mathrm{H} 1 \mathrm{~A}-\mathrm{C} 1-\mathrm{H} 1 \mathrm{~B}$ & 108.2 & $\mathrm{C} 12-\mathrm{C} 13-\mathrm{H} 13$ & 119.4 \\
\hline $\mathrm{O} 1-\mathrm{C} 2-\mathrm{C} 1$ & $111.87(16)$ & $\mathrm{C} 8-\mathrm{C} 13-\mathrm{H} 13$ & 119.4 \\
\hline $\mathrm{O} 1-\mathrm{C} 2-\mathrm{H} 2 \mathrm{~A}$ & 109.2 & $\mathrm{C} 19-\mathrm{C} 14-\mathrm{C} 15$ & $117.86(17)$ \\
\hline $\mathrm{C} 1-\mathrm{C} 2-\mathrm{H} 2 \mathrm{~A}$ & 109.2 & $\mathrm{C} 19-\mathrm{C} 14-\mathrm{C} 7$ & $122.03(17)$ \\
\hline $\mathrm{O} 1-\mathrm{C} 2-\mathrm{H} 2 \mathrm{~B}$ & 109.2 & $\mathrm{C} 15-\mathrm{C} 14-\mathrm{C} 7$ & $120.10(17)$ \\
\hline $\mathrm{C} 1-\mathrm{C} 2-\mathrm{H} 2 \mathrm{~B}$ & 109.2 & $\mathrm{C} 16-\mathrm{C} 15-\mathrm{C} 14$ & $121.60(17)$ \\
\hline $\mathrm{H} 2 \mathrm{~A}-\mathrm{C} 2-\mathrm{H} 2 \mathrm{~B}$ & 107.9 & $\mathrm{C} 16-\mathrm{C} 15-\mathrm{H} 15$ & 119.2 \\
\hline $\mathrm{O} 1-\mathrm{C} 3-\mathrm{C} 4$ & $111.29(16)$ & $\mathrm{C} 14-\mathrm{C} 15-\mathrm{H} 15$ & 119.2 \\
\hline
\end{tabular}




\begin{tabular}{|c|c|c|c|}
\hline $\mathrm{O} 1-\mathrm{C} 3-\mathrm{H} 3 \mathrm{~A}$ & 109.4 & $\mathrm{O} 3-\mathrm{C} 16-\mathrm{C} 15$ & $124.38(17)$ \\
\hline $\mathrm{C} 4-\mathrm{C} 3-\mathrm{H} 3 \mathrm{~A}$ & 109.4 & $\mathrm{O} 3-\mathrm{C} 16-\mathrm{C} 17$ & $115.68(17)$ \\
\hline $\mathrm{O} 1-\mathrm{C} 3-\mathrm{H} 3 \mathrm{~B}$ & 109.4 & $\mathrm{C} 15-\mathrm{C} 16-\mathrm{C} 17$ & $119.94(17)$ \\
\hline $\mathrm{C} 4-\mathrm{C} 3-\mathrm{H} 3 \mathrm{~B}$ & 109.4 & $\mathrm{O} 4-\mathrm{C} 17-\mathrm{C} 18$ & $125.23(17)$ \\
\hline $\mathrm{H} 3 \mathrm{~A}-\mathrm{C} 3-\mathrm{H} 3 \mathrm{~B}$ & 108.0 & $\mathrm{O} 4-\mathrm{C} 17-\mathrm{C} 16$ & $115.56(17)$ \\
\hline $\mathrm{N} 1-\mathrm{C} 4-\mathrm{C} 3$ & $110.16(16)$ & $\mathrm{C} 18-\mathrm{C} 17-\mathrm{C} 16$ & $119.21(18)$ \\
\hline $\mathrm{N} 1-\mathrm{C} 4-\mathrm{H} 4 \mathrm{~A}$ & 109.6 & $\mathrm{C} 17-\mathrm{C} 18-\mathrm{C} 19$ & $120.49(18)$ \\
\hline $\mathrm{C} 3-\mathrm{C} 4-\mathrm{H} 4 \mathrm{~A}$ & 109.6 & $\mathrm{C} 17-\mathrm{C} 18-\mathrm{H} 18$ & 119.8 \\
\hline $\mathrm{N} 1-\mathrm{C} 4-\mathrm{H} 4 \mathrm{~B}$ & 109.6 & $\mathrm{C} 19-\mathrm{C} 18-\mathrm{H} 18$ & 119.8 \\
\hline $\mathrm{C} 3-\mathrm{C} 4-\mathrm{H} 4 \mathrm{~B}$ & 109.6 & $\mathrm{C} 14-\mathrm{C} 19-\mathrm{C} 18$ & $120.89(18)$ \\
\hline $\mathrm{H} 4 \mathrm{~A}-\mathrm{C} 4-\mathrm{H} 4 \mathrm{~B}$ & 108.1 & $\mathrm{C} 14-\mathrm{C} 19-\mathrm{H} 19$ & 119.6 \\
\hline $\mathrm{O} 2-\mathrm{C} 5-\mathrm{N} 1$ & $121.99(18)$ & $\mathrm{C} 18-\mathrm{C} 19-\mathrm{H} 19$ & 119.6 \\
\hline $\mathrm{O} 2-\mathrm{C} 5-\mathrm{C} 6$ & $121.71(17)$ & $\mathrm{O} 3-\mathrm{C} 20-\mathrm{H} 20 \mathrm{~A}$ & 109.5 \\
\hline $\mathrm{N} 1-\mathrm{C} 5-\mathrm{C} 6$ & $116.24(16)$ & $\mathrm{O} 3-\mathrm{C} 20-\mathrm{H} 20 \mathrm{~B}$ & 109.5 \\
\hline $\mathrm{C} 7-\mathrm{C} 6-\mathrm{C} 5$ & $126.17(17)$ & $\mathrm{H} 20 \mathrm{~A}-\mathrm{C} 20-\mathrm{H} 20 \mathrm{~B}$ & 109.5 \\
\hline $\mathrm{C} 7-\mathrm{C} 6-\mathrm{H} 6$ & 116.9 & $\mathrm{O} 3-\mathrm{C} 20-\mathrm{H} 20 \mathrm{C}$ & 109.5 \\
\hline $\mathrm{C} 5-\mathrm{C} 6-\mathrm{H} 6$ & 116.9 & $\mathrm{H} 20 \mathrm{~A}-\mathrm{C} 20-\mathrm{H} 20 \mathrm{C}$ & 109.5 \\
\hline $\mathrm{C} 6-\mathrm{C} 7-\mathrm{C} 14$ & $122.97(18)$ & $\mathrm{H} 20 \mathrm{~B}-\mathrm{C} 20-\mathrm{H} 20 \mathrm{C}$ & 109.5 \\
\hline $\mathrm{C} 6-\mathrm{C} 7-\mathrm{C} 8$ & $118.35(17)$ & $\mathrm{O} 4-\mathrm{C} 21-\mathrm{H} 21 \mathrm{~A}$ & 109.5 \\
\hline $\mathrm{C} 14-\mathrm{C} 7-\mathrm{C} 8$ & $118.52(16)$ & $\mathrm{O} 4-\mathrm{C} 21-\mathrm{H} 21 \mathrm{~B}$ & 109.5 \\
\hline $\mathrm{C} 9-\mathrm{C} 8-\mathrm{C} 13$ & $118.25(18)$ & $\mathrm{H} 21 \mathrm{~A}-\mathrm{C} 21-\mathrm{H} 21 \mathrm{~B}$ & 109.5 \\
\hline $\mathrm{C} 9-\mathrm{C} 8-\mathrm{C} 7$ & $121.42(17)$ & $\mathrm{O} 4-\mathrm{C} 21-\mathrm{H} 21 \mathrm{C}$ & 109.5 \\
\hline $\mathrm{C} 13-\mathrm{C} 8-\mathrm{C} 7$ & $120.18(17)$ & $\mathrm{H} 21 \mathrm{~A}-\mathrm{C} 21-\mathrm{H} 21 \mathrm{C}$ & 109.5 \\
\hline $\mathrm{C} 10-\mathrm{C} 9-\mathrm{C} 8$ & $120.9(2)$ & $\mathrm{H} 21 \mathrm{~B}-\mathrm{C} 21-\mathrm{H} 21 \mathrm{C}$ & 109.5 \\
\hline $\mathrm{C} 10-\mathrm{C} 9-\mathrm{H} 9$ & 119.5 & & \\
\hline $\mathrm{C} 5-\mathrm{N} 1-\mathrm{C} 1-\mathrm{C} 2$ & $132.71(19)$ & $\mathrm{C} 11-\mathrm{C} 11-\mathrm{C} 12-\mathrm{C} 13$ & $-179.22(15)$ \\
\hline $\mathrm{C} 4-\mathrm{N} 1-\mathrm{C} 1-\mathrm{C} 2$ & $-51.7(2)$ & $\mathrm{C} 11-\mathrm{C} 12-\mathrm{C} 13-\mathrm{C} 8$ & $1.9(3)$ \\
\hline $\mathrm{C} 3-\mathrm{O} 1-\mathrm{C} 2-\mathrm{C} 1$ & $-60.0(2)$ & $\mathrm{C} 9-\mathrm{C} 8-\mathrm{C} 13-\mathrm{C} 12$ & $-2.9(3)$ \\
\hline $\mathrm{N} 1-\mathrm{C} 1-\mathrm{C} 2-\mathrm{O} 1$ & $55.2(2)$ & $\mathrm{C} 7-\mathrm{C} 8-\mathrm{C} 13-\mathrm{C} 12$ & $172.68(17)$ \\
\hline $\mathrm{C} 2-\mathrm{O} 1-\mathrm{C} 3-\mathrm{C} 4$ & $59.7(2)$ & $\mathrm{C} 6-\mathrm{C} 7-\mathrm{C} 14-\mathrm{C} 19$ & $-139.6(2)$ \\
\hline $\mathrm{C} 5-\mathrm{N} 1-\mathrm{C} 4-\mathrm{C} 3$ & $-132.19(19)$ & $\mathrm{C} 8-\mathrm{C} 7-\mathrm{C} 14-\mathrm{C} 19$ & $45.1(2)$ \\
\hline $\mathrm{C} 1-\mathrm{N} 1-\mathrm{C} 4-\mathrm{C} 3$ & $52.0(2)$ & $\mathrm{C} 6-\mathrm{C} 7-\mathrm{C} 14-\mathrm{C} 15$ & $41.5(3)$ \\
\hline $\mathrm{O} 1-\mathrm{C} 3-\mathrm{C} 4-\mathrm{N} 1$ & $-55.3(2)$ & $\mathrm{C} 8-\mathrm{C} 7-\mathrm{C} 14-\mathrm{C} 15$ & $-133.79(18)$ \\
\hline $\mathrm{C} 4-\mathrm{N} 1-\mathrm{C} 5-\mathrm{O} 2$ & $8.1(3)$ & $\mathrm{C} 19-\mathrm{C} 14-\mathrm{C} 15-\mathrm{C} 16$ & $0.5(3)$ \\
\hline $\mathrm{C} 1-\mathrm{N} 1-\mathrm{C} 5-\mathrm{O} 2$ & $-176.63(17)$ & $\mathrm{C} 7-\mathrm{C} 14-\mathrm{C} 15-\mathrm{C} 16$ & $179.42(16)$ \\
\hline $\mathrm{C} 4-\mathrm{N} 1-\mathrm{C} 5-\mathrm{C} 6$ & $-169.19(17)$ & $\mathrm{C} 20-\mathrm{O} 3-\mathrm{C} 16-\mathrm{C} 15$ & $-2.2(3)$ \\
\hline $\mathrm{C} 1-\mathrm{N} 1-\mathrm{C} 5-\mathrm{C} 6$ & $6.1(3)$ & $\mathrm{C} 20-\mathrm{O} 3-\mathrm{C} 16-\mathrm{C} 17$ & $178.19(16)$ \\
\hline $\mathrm{O} 2-\mathrm{C} 5-\mathrm{C} 6-\mathrm{C} 7$ & $50.0(3)$ & $\mathrm{C} 14-\mathrm{C} 15-\mathrm{C} 16-\mathrm{O} 3$ & $-179.46(17)$ \\
\hline $\mathrm{N} 1-\mathrm{C} 5-\mathrm{C} 6-\mathrm{C} 7$ & $-132.7(2)$ & $\mathrm{C} 14-\mathrm{C} 15-\mathrm{C} 16-\mathrm{C} 17$ & $0.2(3)$ \\
\hline $\mathrm{C} 5-\mathrm{C} 6-\mathrm{C} 7-\mathrm{C} 14$ & $9.2(3)$ & $\mathrm{C} 21-\mathrm{O} 4-\mathrm{C} 17-\mathrm{C} 18$ & $-8.5(3)$ \\
\hline $\mathrm{C} 5-\mathrm{C} 6-\mathrm{C} 7-\mathrm{C} 8$ & $-175.53(17)$ & $\mathrm{C} 21-\mathrm{O} 4-\mathrm{C} 17-\mathrm{C} 16$ & $171.23(17)$ \\
\hline $\mathrm{C} 6-\mathrm{C} 7-\mathrm{C} 8-\mathrm{C} 9$ & $37.0(3)$ & $\mathrm{O} 3-\mathrm{C} 16-\mathrm{C} 17-\mathrm{O} 4$ & $-0.4(2)$ \\
\hline $\mathrm{C} 14-\mathrm{C} 7-\mathrm{C} 8-\mathrm{C} 9$ & $-147.52(18)$ & $\mathrm{C} 15-\mathrm{C} 16-\mathrm{C} 17-\mathrm{O} 4$ & $179.97(16)$ \\
\hline $\mathrm{C} 6-\mathrm{C} 7-\mathrm{C} 8-\mathrm{C} 13$ & $-138.47(19)$ & $\mathrm{O} 3-\mathrm{C} 16-\mathrm{C} 17-\mathrm{C} 18$ & $179.34(16)$ \\
\hline $\mathrm{C} 14-\mathrm{C} 7-\mathrm{C} 8-\mathrm{C} 13$ & $37.0(2)$ & $\mathrm{C} 15-\mathrm{C} 16-\mathrm{C} 17-\mathrm{C} 18$ & $-0.3(3)$ \\
\hline $\mathrm{C} 13-\mathrm{C} 8-\mathrm{C} 9-\mathrm{C} 10$ & $1.6(3)$ & $\mathrm{O} 4-\mathrm{C} 17-\mathrm{C} 18-\mathrm{C} 19$ & $179.47(17)$ \\
\hline $\mathrm{C} 7-\mathrm{C} 8-\mathrm{C} 9-\mathrm{C} 10$ & $-173.99(18)$ & $\mathrm{C} 16-\mathrm{C} 17-\mathrm{C} 18-\mathrm{C} 19$ & $-0.2(3)$ \\
\hline
\end{tabular}




$\begin{array}{llll}\mathrm{C} 8-\mathrm{C} 9-\mathrm{C} 10-\mathrm{C} 11 & 0.8(3) & \mathrm{C} 15-\mathrm{C} 14-\mathrm{C} 19-\mathrm{C} 18 & -1.0(3) \\ \mathrm{C} 9-\mathrm{C} 10-\mathrm{C} 11-\mathrm{C} 12 & -1.8(3) & \mathrm{C} 7-\mathrm{C} 14-\mathrm{C} 19-\mathrm{C} 18 & -179.93(17) \\ \mathrm{C} 9-\mathrm{C} 10-\mathrm{C} 11-\mathrm{C} 11 & 177.89(16) & \mathrm{C} 17-\mathrm{C} 18-\mathrm{C} 19-\mathrm{C} 14 & 0.9(3) \\ \mathrm{C} 10-\mathrm{C} 11-\mathrm{C} 12-\mathrm{C} 13 & 0.5(3) & & \end{array}$

Hydrogen-bond geometry $\left(\AA,{ }^{\circ}\right)$

\begin{tabular}{lllll}
\hline$D-\mathrm{H}^{\cdots} A$ & $D-\mathrm{H}$ & $\mathrm{H} \cdots A$ & $D \cdots A$ & $D-\mathrm{H} \cdots A$ \\
\hline $\mathrm{C} 1-\mathrm{H} 1 B \cdots \mathrm{O} 2^{\mathrm{i}}$ & 0.99 & 2.53 & $3.167(2)$ & 122 \\
$\mathrm{C} 13-\mathrm{H} 13 \cdots \mathrm{O} 2^{\mathrm{ii}}$ & 0.95 & 2.38 & $3.166(2)$ & 140 \\
$\mathrm{C} 20-\mathrm{H} 20 B \cdots \mathrm{O} 1^{\mathrm{iii}}$ & 0.98 & 2.64 & $3.010(2)$ & 103
\end{tabular}

Symmetry codes: (i) $x+1, y, z$; (ii) $-x, y+1 / 2,-z+3 / 2$; (iii) $-x+1,-y+1,-z+2$. 